\title{
Hétérographies du désir
}

Pratiques votives au monastère de Saint-Georges (Büyükada, Istanbul)

\section{Benoît Fliche et Manoël Pénicaud}

\section{CpenEdition}

Journals

Édition électronique

URL : https://journals.openedition.org/tc/9766

DOI : $10.4000 /$ tc. 9766

ISSN : 1952-420X

Éditeur

Éditions de l'EHESS

\section{Édition imprimée}

Date de publication : 30 octobre 2018

Pagination : 142-161

ISBN : 2-7132-2751-6

ISSN : 0248-6016

Référence électronique

Benoît Fliche et Manoël Pénicaud, « Hétérographies du désir », Techniques \& Culture [En ligne], 70 |

2018, mis en ligne le 06 décembre 2020, consulté le 29 septembre 2022. URL : http://

journals.openedition.org/tc/9766 ; DOI : https://doi.org/10.4000/tc.9766 


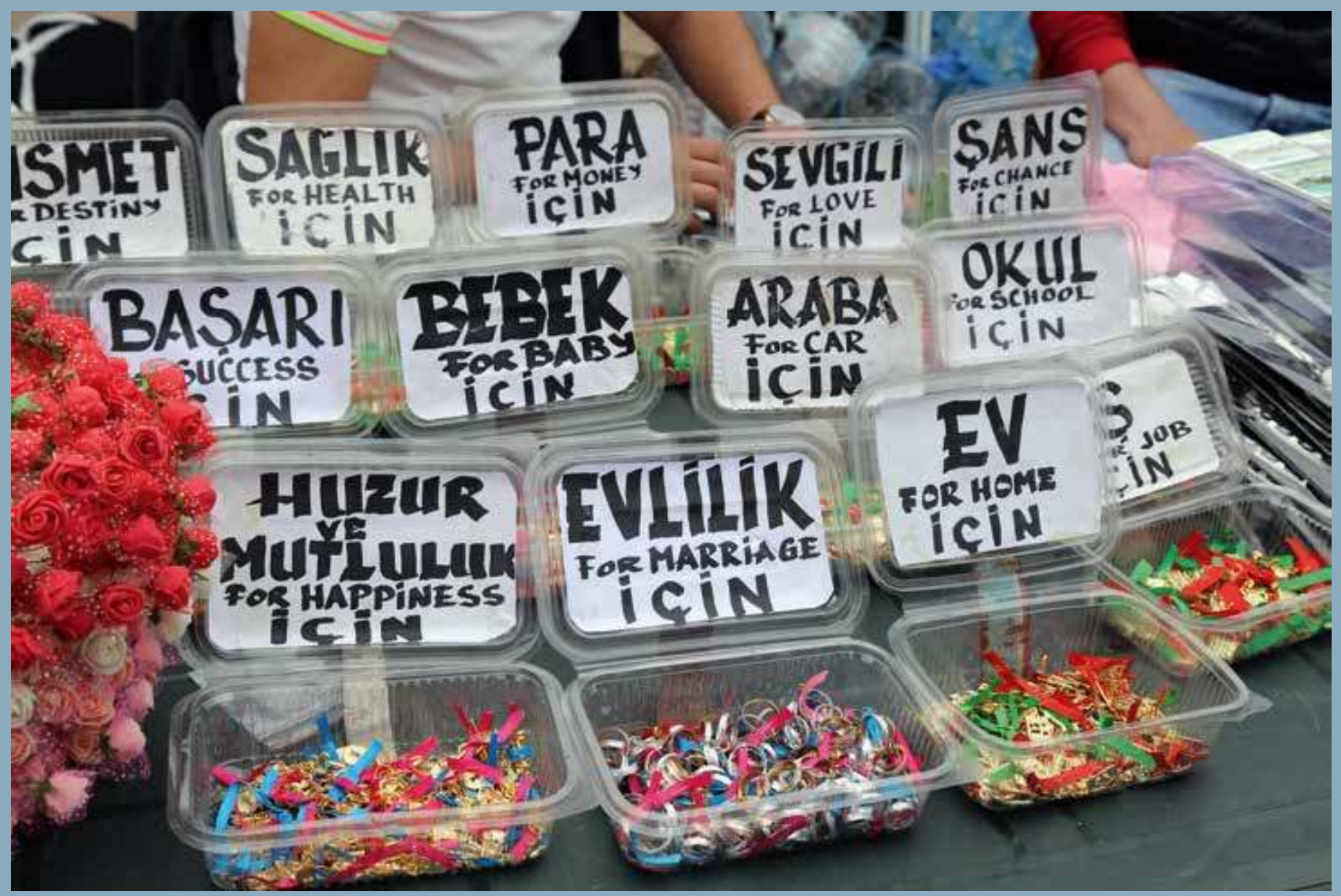




\section{Hétérographies du désir Pratiques votives au monastère de Saint-Georges (Büyükada, Istanbul)}

Le 23 avril n'est pas seulement la fête des enfants et de la souveraineté nationale qui commémore les prémices de la République turque fondée par Mustapha Kemal Atatürk. Ce jour férié correspond aussi à la fête de saint Georges, figure majeure dans le christianisme oriental. Ce matin-là, les innombrables embarcadères d'Istanbul sont pris d'assaut par des dizaines de milliers de personnes. Toutes souhaitent atteindre l'île de Büyükada, la plus grande de l'archipel des Princes (en grec, Prinkipo), au sommet de laquelle s'élève un monastère grec orthodoxe qui abrite une icône miraculeuse de saint Georges. Une heure plus tard, les bateaux déversent une foule immense de pèlerins qui envahissent le petit port touristique. Les voitures étant interdites sur l'île, certains cherchent à prendre une calèche, tandis que la plupart montent à pied vers le monastère. Sur la place de l'Union (Birlik Meydanı) règne une foire typique des pèlerinages: lieu de retrouvailles, marché d'échoppes éphémères, carrefour incessant des calèches, sous le contrôle discret des autorités. C'est là que débute le chemin pédestre du sanctuaire qui semble chargé d'une force spirituelle particulièrement dense ce jour-là, tout au moins dans les représentations des pèlerins.

La grande spécificité de ce jour de fête est que la grande majorité des visiteurs ne sont pas chrétiens. En effet, le 23 avril, ce lieu saint attire en masse des musulmans. Ou plutôt faudrait-il dire «musulmanes», car la balance des sexes est de l'ordre de deux femmes pour un homme sur le ponton d'arrivée, pour être ensuite de dix pour un à l'intérieur du sanctuaire; $89 \%$ des visiteurs sont des femmes non voilées ce qui, aujourd'hui en Turquie, n'est pas sans signification puisqu'environ $70 \%$ des femmes déclarent porter le voile dans les sondages. $1 \%$ des visiteurs sont des femmes portant le voile. Les enfants sont assez rares. Certes, cette abondante fréquentation musulmane peut paraître inopinée ou incongrue de prime abord, mais elle s'inscrit en fait dans un phénomène plus vaste, tant dans la longue durée que sur le plan géographique: la visite des sanctuaires de l'«autre religieux». Cela a bien été documenté dès la fin de l'Empire Ottoman par Frederick Hasluck (Hasluck 2000), avant qu'un nombre croissant de travaux 
récents s'intéressent à ces franchissements temporaires de la frontière religieuse (Albera \& Couroucli 2009, Albera \& Pénicaud 2017, Barkan \& Barkey 2014, Farra-Haddad 2015, Fliche 2014, Hobart \& Zarcone 2017, etc.) ${ }^{1}$.

Par son dynamisme et son ampleur quantitative concentrés sur deux journées par an, ce pèlerinage "partagé » est sans doute l'un des plus importants du Moyen-Orient. Aya Yorgi (saint Georges, en grec, appellation utilisée par les Turcs) est aussi l'un des sanctuaires les plus fréquentés d'Istanbul2. Il s'inscrit dans une géographie du sacré plus large qui ne se limite pas seulement aux églises et sources sacrées chrétiennes (ayazma), mais compte aussi de nombreux mausolées de saints musulmans (türbe) et de lieux sacrés (yatır) chargés de grâce. Aya Yorgi est victime de son succès ${ }^{3}$. La conjugaison de la médiatisation, du bouche-à-oreille et de la concordance avec un jour férié, a pleinement propulsé le succès de ce pèlerinage. La foule est croissante d'année en année. En 2014, l'affluence aurait dépassé les 75000 personnes alors que dix ans auparavant nous pouvions compter environ 15000 à 20000 personnes.

Il s'agit en outre du pèlerinage où le désir votif se matérialise plus que dans tout autre sanctuaire en Turquie. Son succès semble à première vue lié à la puissance votive qui s'en dégagerait: les pèlerins y vont parce qu'ils espèrent que leurs vœux seront exaucés, comme dans la plupart des lieux saints. L'efficacité performative y est primordiale. À l'entour du monastère, l'espace est envahi par une matérialité votive débordante caractérisée par son hétérogénéité formelle (messages écrits, dessins, «sculptures», etc.). Comment expliquer une telle créativité rituelle à l'endroit des écritures votives? Nous proposons de définir comme «hétérographies » ces façons plurielles d'inscrire un rapport sensible au monde et au divin, dans une élaboration iconographique éphémère ou de signes qui tiennent de la métonymie (Albera 2013). En d'autres termes, l'hypothèse centrale est que l'écriture votive au sens large ("hétérographie ») crée de l'esthétique qui détermine, en partie, l'expérience religieuse.

S'agissant de musulmans, ces praxeis constituent autant d'exopraxeis puisqu'ils empruntent à la fois le lieu et certaines pratiques de l'autre religieux, générant des braconnages et des bricolages transfrontaliers des plus inventifs (Fliche 2010: 89). Comment la démultiplication de ces hétérographies votives exoprates ${ }^{4}$ bouleverse-t-elle l'écosystème de ce pèlerinage, opérant un renversement discret de la croyance qui structurait jusqu'à présent sa topographie? Plus largement, on questionnera le rapport au monde et au divin que traduisent les hétérographies votives: quelle est la place de saint Georges et de Dieu? Quel type de causalité est en jeu dans le désir votif?

Sur le plan méthodologique, cette ethnographie à quatre mains s'appuie sur une dizaine d'années d'observation pour saisir les dynamiques qui traversent et modifient la chorégraphie rituelle du pèlerinage. Cet article repose en outre sur l'analyse d'un large corpus de vœux écrits photographiés sur place. On se basera aussi sur des entretiens livrant le point de vue pragmatique des acteurs et des moines grecs qui administrent ce «lieu pratiqué», selon la formule de Michel de Certeau (Certeau 1990). Enfin, mentionnons l'apport déterminant de l'anthropologie visuelle (photographique et filmique), particulièrement adéquate pour capturer in vivo ce foisonnement votif et pour mieux l'appréhender rétrospectivement (Pénicaud 2014b). La photographie est ici envisagée comme une écriture ethnographique en soi. 


\section{Ethnographie d'un pèlerinage exoprate}

\section{Miracles, guérisons et démarches votives}

Vraisemblablement construit au xe siècle, le monastère de Saint-Georges fait l'objet de plusieurs légendes locales liées à l'icône miraculeuse du saint. Comme ailleurs, le miracle est fondateur. Sans remonter au Moyen Âge ${ }^{5}$, c'est à la suite d'une guérison retentissante que le pèlerinage prit son ampleur actuelle: un homme aurait guéri d'un cancer incurable après avoir accroché sa montre contre l'icône sertie d'argent, si bien qu'aujourd'hui, de nombreux pèlerins répètent ce geste.

Du thème de la guérison, émerge donc celui de la démarche votive (dilek, en turc), au cour de ce pèlerinage. Si les visiteurs musulmans semblent libres de se comporter à leur guise, les moines grecs tentent de réguler l'effervescence créatrice, notamment par des panneaux indiquant: «Attention. Il est péché [günah] d'écrire dans l'enceinte de l'église. S'il vous plaît, n'écrivez pas sur les icônes [avec des bougies] ni sur les murs. Il y a une boîte à voux dans l'église! Écrivez-les sur un papier et déposez-les dans la boîte à vœux. Nous vous remercions. » Mais ces injonctions à une « orthodoxie» restent lettre morte. Les moines ne peuvent pas encadrer la foule, ni faire respecter les règles qui prévalent le reste du temps. Le 23 avril, tout est inversé. Les interdictions vestimentaires, de filmer, de photographier ou même de parler dans l'église tombent. Par ailleurs, les visiteurs venant pour la première fois n'ont pas pu intérioriser les normes de bonne conduite. De façon générale, la norme de l'« orthodoxie (voie droite) est balayée par le flux des pratiques «hétérodoxes».

À ce stade, décrivons la forte hétérogénéité de la matérialité votive. Outre les messages textuels, on compte pléthore de dessins, de cierges de couleurs, de bobines de fils multicolores, de sculptures de brindilles ou de cailloux, de pièces de monnaie incrustées dans la cire brûlante, etc. Cette variété des formes et des matières constitue dès lors une palette d'hétérographies votives. Ces écritures - au sens large du terme - votives et protéiformes révèlent en fait le rapport au monde et au divin des agents. Ayant recours à une panoplie d'actions (gestes) et de supports (objets), ils écrivent leur désir intime dans un style qui est finalement relativement libre puisqu'il se nourrit abondamment d'improvisation et d'imitation qui ne se soucient pas particulièrement du regard d'autrui. L'espace étant a priori libre de droit, on «fait avec » ce dont on dispose comme accessoire in situ pour matérialiser son vœu (Certeau 1990). 


\section{Dimension foraine en aval du monastère}

L'espace-temps du pèlerinage commence en dehors du sanctuaire et débute sur la place de l'Union déjà mentionnée, au pied de la colline. S'y étend un marché où des stands éclosent la veille du 23 avril et du 24 septembre. Comme l'on sait, la foire constitue un pilier du phénomène pèlerin en général (Dupront 1987, Reader 2014). Dans les échoppes éphémères est vendue une grande part de l'attirail votif des pèlerins. Citons d'abord ce qui relève du lokma, c'est-à-dire de l'offrande, à travers les boîtes de sucre conduisant à un rituel déterminant: les gens exaucés à la Sainte-Thècle reviennent à la Saint-Georges et vice versa. Ils doivent monter en silence à l'église et distribuer à sa sortie des carreaux de sucre sans souffler mot. À côté trônent les bouteilles d'huile pour les offrandes au monastère. Puis viennent les objets votifs, comme les cierges: à chaque couleur correspondent une attente et un usage précis (santé, mariage, enfant, travail, etc.). Il en va de même avec les amulettes: des anneaux enchevêtrés pour le mariage, un fer à cheval avec un œil (boncuk) contre le mauvais sort (nazar), un grelot pour la chance, une pièce de monnaie pour l'argent, un livre pour la réussite scolaire, une maison pour l'accès à la propriété, une clef pour la prospérité, un cœur pour l'amour, une clef et un cadenas pour le destin (kismet), etc. Le répertoire s'est enrichi puisqu'entre 2006 et 2014, nous sommes passés de 12 à 27 types d'amulettes, avec désormais des items concernant le service militaire, le handicap, le chagrin, la sérénité, la liberté, et dernièrement contre la magie. Le champ sémantique du vœu s'est considérablement étoffé.

Ces médailles, les gens les accrochent à un arbre, à leur vêtement, ou bien les rapportent chez eux, quand d'autres disent qu'il faut les jeter à la mer une fois le vœu accompli. Il semble qu'il n'y ait pas de règle en la matière. Cette spécialisation croissante de l'offre est une innovation qui répond à la demande des agents tout en leur suggérant - dans une intention commerciale - de nouvelles perspectives votives.

\section{Rituel des fils votifs}

Les marchands proposent aussi des bobines de fil qui servent à l'accomplissement du rituel votif le plus emblématique. Sur le chemin, beaucoup de femmes attachent ce fil à un arbuste et déroulent ensuite la bobine en silence et en formulant leur vœu intérieurement. «Le fait de monter jusqu'ici, c'est un peu comme s'écouter soi-même. Le vœu de silence pendant l'ascension favorise cette écoute de soi. Les gens ont besoin de faire ce type de rituels pour se retrouver face à eux-mêmes», déclare une jeune informatrice musulmane. Cette pratique a été combattue, en vain, tant par les moines que les services municipaux. Depuis 2012, dès le matin, le chemin ressemble à une machine à tisser de fils multicolores. En fin de journée, ce sont de vastes toiles d'araignées de l'espoir qui se déploient, dans lesquelles tant de visiteuses semblent prisonnières, la main tenant le précieux fil d'Ariane, absorbées dans l'intériorité de leur désir. 


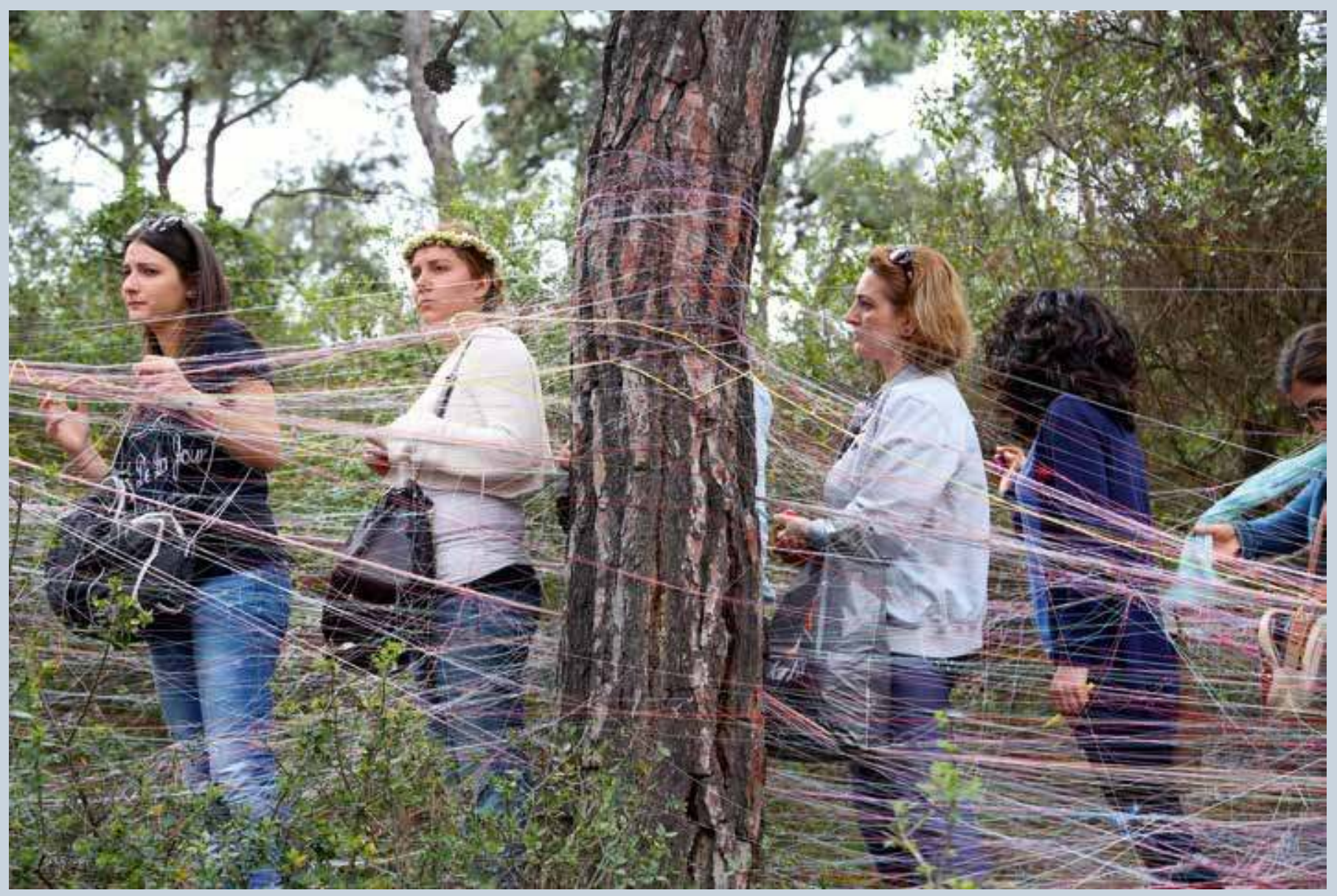




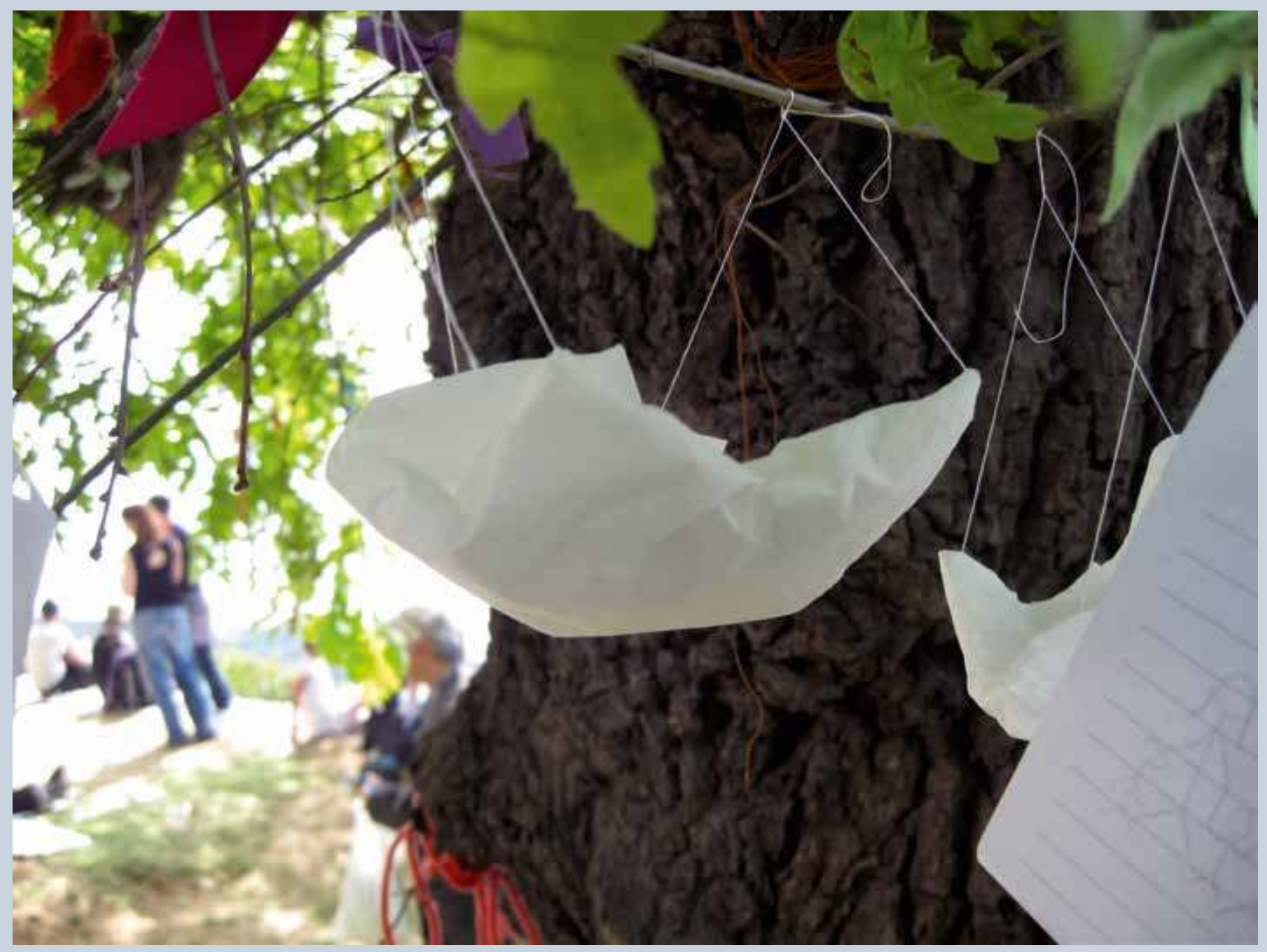

2. Vœux en forme de berceaux

Une pierre à l'intérieur matérialise l'enfant désiré. 
Arrivées à destination, elles finissent de dérouler la totalité de leur bobine qu'elles réenroulent sur les arbres environnants, certaines croyant que le vou se réalisera si le fil est sans cassure, alors que d'autres estiment que le fil matérialise le temps qui s'écoule entre le vœu et sa réalisation, si bien qu'une cassure en réduit l'attente. Accrochés aux branches des arbres se trouvent les premiers bouts de tissus, les premiers vœux épinglés avec minutie. Soulignons aussi un type de vœu particulièrement touchant, prenant la forme métaphorique d'un berceau, avec une pierre à l'intérieur pour incarner l'enfant désiré.

En chemin, on croise aussi des groupes de chrétiens évangéliques cherchant à convertir, et ce, sans crainte des autorités, alors que tout acte de prosélytisme est strictement condamné par la loi. Leur recours à de la musique live attire la curiosité des pèlerins qui se voient distribuer des brochures sur Jésus ou bien participer à une session d'imposition des mains. Souvent constitués d'Asiatiques, ces groupes se rapprochent du monastère d'année en année, suscitant le courroux des moines ${ }^{6}$.

À l'entrée du monastère, la foule forme une file d'attente compacte. «Rien que pour la fête de saint Georges, il y a une moyenne de 70000 personnes en une journée. C'est une journée vraiment spéciale, ça dépasse l'imagination! Parfois, les gens attendent deux ou trois heures, ce n'est pas si simple d'entrer dans l'église de Saint-Georges», expliquait en 2014 le moine grec Ézéchiel. Des barrières encadrent ce flux ininterrompu de ferveur, sous le contrôle des forces de sécurité.

\section{Une effervescence rituelle incontrôlable}

L'intérieur de l'église est occupé dès son ouverture à l'aube, bien avant le début de l'office. À peine entrés, les pèlerins brûlent des cierges. Il n'y a qu'un sens de circulation et l'on est canalisé vers la sortie, surtout pendant la messe. Seuls les plus fervents parviennent à se rapprocher du chœur où les trois moines officient. «La plupart des gens qui viennent ici ne sont pas chrétiens. Ils allument des bougies, et quand ils se trouvent dans l'église, même s'ils n'avaient pas prévu de prier, ils finissent par prier ou faire des vœux», rapporte Ézéchiel. Au-delà de la prière des musulmans rendue visible par le geste des mains ouvertes, paumes en l'air avant de les passer sur le visage, les vœux textuels représentent le rituel le plus important dans l'église. Cela est certes assez banal d'écrire son vœu ou une intention de prière dans le monde chrétien (Lamireau 2009), mais cela se complexifie quand il s'agit de musulmans dans un lieu chrétien, ce qui a notamment été analysé ailleurs à Istanbul (Albera \& Fliche 2009, Fliche 2014).

Au fond de l'église, trône la boîte à vœux (dilek kutusu) qui reçoit des dizaines de messages par minute. Plusieurs fois par jour, les moines la vident, après quoi ils sont censés prier pour chaque demande avant de brûler rituellement ces feuillets. En 2014, le même Ézéchiel confiait: «Parfois, les vœux sont très basiques, par exemple que Dieu aide mes enfants à réussir les examens à l'université. Mais ces vœux peuvent être très sérieux: que Dieu guérisse mon enfant malade du cancer. D'autres fois, des enfants demandent que leurs parents arrêtent de boire ou 
qu'ils se réconcilient après une séparation. » Avant les années 2010, tous les messages étaient déposés dans la boîte puisque, bénis, ils étaient susceptibles d'être exaucés. Mais depuis, force est d'observer un débordement de l'écrit votif: les papiers sont désormais glissés partout dans l'église, sous les icônes, voire dans la cour du monastère ${ }^{7}$. La sainteté est «officiellement» incarnée par l'icône miraculeuse, protégée sous verre et cadenassée. Seuls les moines peuvent insérer un ex-voto à son contact, ce qui nécessite une médiation soutenue.

La densité de la foule fait que l'on se retrouve vite en dehors de l'église, où sont distribués les morceaux de sucre déjà évoqués. Si certains pèlerins en mangent, d'autres s'en servent comme nouveau matériau votif en l'insérant dans l'écorce rugueuse d'un grand arbre qui devient parsemé de centaines de points blancs. Beaucoup réutilisent ces carreaux de sucre pour dessiner leurs vœux sur une margelle (maison, cœur, bébé, etc.). Le vœu fonctionne dans une logique iconique forte.

En contrebas, une enfilade d'arbres fruitiers devient une station quasi-obligée. Presque chaque visiteur y accroche un bout de tissu, un mouchoir en papier ou bien une petite amulette achetée au marché. Plus loin, sur un terre-plein, on compose à nouveau des figures à partir de matériaux végétaux (brindilles, cailloux), de cire et de tout ce que l'on a sous la main. La fin du parcours intra-muros est marquée par le passage d'un tourniquet impossible à franchir en sens inverse. Mais cela ne marque pas pour autant la fin de la performance votive qui continue à l'extérieur de l'enceinte, à commencer par le dépôt continuel d'innombrables cierges colorés dont la cire sert à fixer des pièces de monnaie sur une paroi rocheuse.

Finalement, ce n'est plus seulement l'intérieur du monastère qui est saturé par cette matérialité rituelle, mais l'ensemble de l'espace environnant. N'importe quel arbre devient support potentiel d'expression votive. À la fin du jour, les moines ont beaucoup à faire pour se débarrasser de cette surabondante production, tandis qu'à l'extérieur, les services municipaux s'activent à nettoyer les environs, notamment la toile d'araignée du chemin parcouru de fils. Une fois les pèlerins redescendus vers les bateaux, leurs souhaits les plus chers ne s'apparentent plus bien souvent qu'à des déchets rituels.

\section{Hétérographies votives et causalités divines}

\section{Bouleversement de la centralité sacrale}

La présentation qui vient d'être faite nous conduit à une première réflexion sur la topographie votive qui, au cours des dix dernières années, s'est considérablement élargie. En effet, les vœux débordent littéralement les murs du monastère pour se déverser dans l'espace environnant, en le saturant de signes, de fils, de papiers, de bougies, de morceaux de sucre. La boîte à vœux 

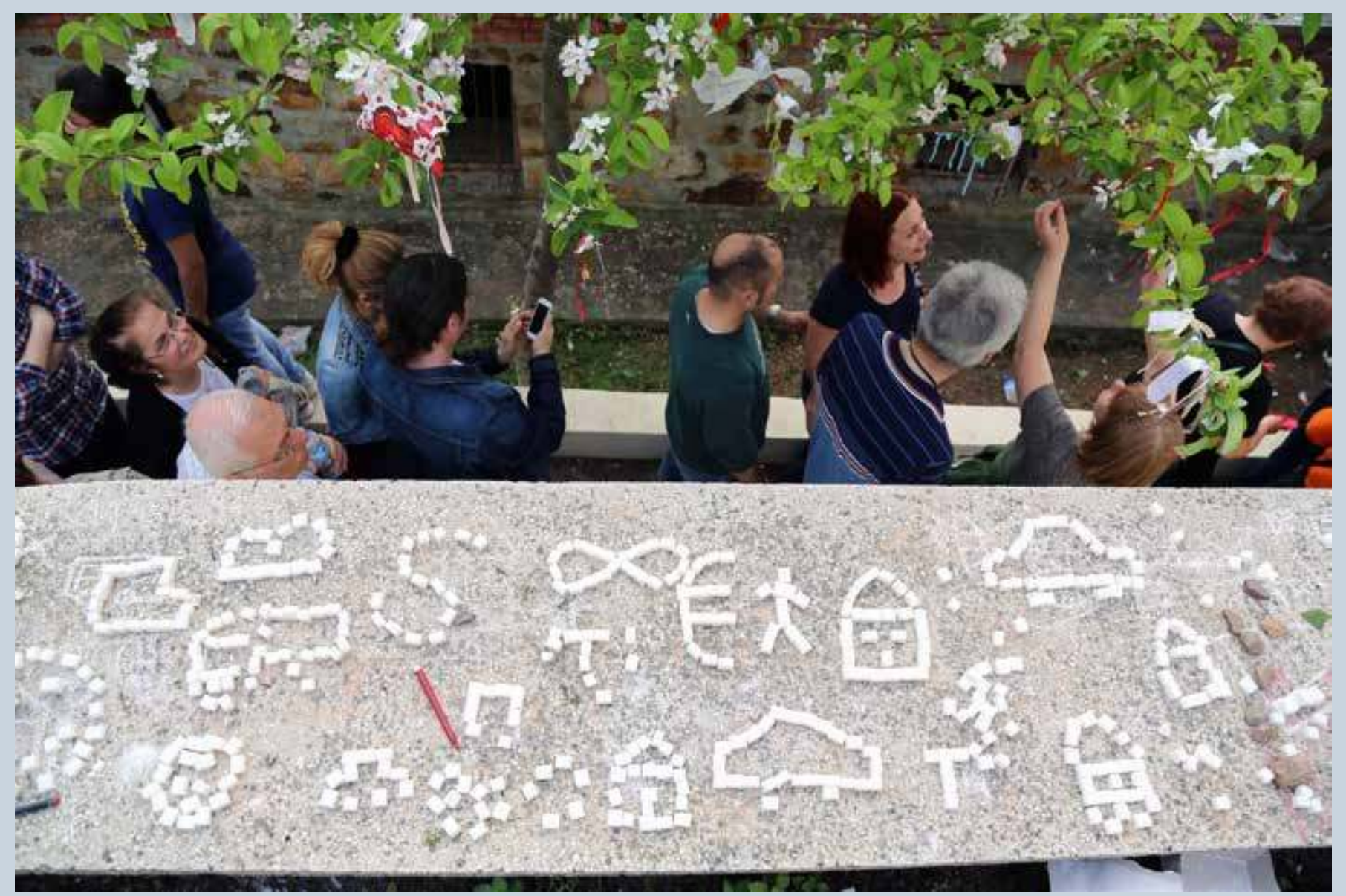

Figures allégoriques Cœurs, maisons, voitures, etc. et arbres à vœux 
n'incarne plus le sacré, ni l'icône, ni l'église que beaucoup ne visitent pas, formulant leurs vœux extra-muros, dans un cercle plus large que le seul sanctuaire. Ce débordement s'explique d'abord par la très grande fréquentation. Sur les 75000 pèlerins estimés en 2014, seule une minorité pénètre dans l'église. Un comptage minutieux effectué au niveau du tourniquet de sortie du monastère, qui n'autorise que les passages individuels, donne une estimation de 20000 visiteurs dans la journée, entre 9 et 18 heures. La plupart des gens restent donc dehors, aux abords du sanctuaire, d'où la prolifération des vœux à l'extérieur.

\section{Bricolage, braconnage et exopraxis}

Ces actes votifs relèvent du «braconnage», que nous entendons comme une jouissance discrète de la coupure, un jeu d'élision sur la différence. Le braconnage est toujours une jouissance d'un différentiel d'espaces, illégitime voire illicite, qui appelle dès lors à la discrétion (Certeau 1990, Fliche 2014). Dans une série d'entretiens réalisés en 2014, l'exopraxis ou l'emprunt du lieu (sacré) de l'autre est pleinement revendiquée. Une jeune musulmane qui vient tous les ans déclare ainsi: «Au final, ici c'est la maison d'Allah. Mosquée, église, ça ne change rien. Grâce à la synergie présente ici, sans utiliser aucun intermédiaire, on envoie directement nos vœux les plus profonds vers l'univers. Tous mes vœux se sont réalisés. Cette année encore, j’ai quelques souhaits à faire et j'ai l'espoir qu'ils se réalisent à leur tour. L'important, c'est de vouloir du fond du cœur et de croire. C'est pour cela que je viens. » À la question de savoir si elle est entrée dans l'église, elle répond: «Bien sûr, je suis entrée, je me suis assise, j’ai fait ma prière, j’ai écrit mes vœux sur un papier que j'ai fait passer sur les icônes avant de le mettre dans la boîte, j’ai prié, j'ai ouvert mon cœur, j'ai cru que mes vœux se réaliseront, j’ai remercié [Dieu] et je suis sortie. » Une autre dame raconte spontanément: «C'est la première fois que j'entre; en ouvrant les mains et en priant Allah puis je suis sortie. Au bout du compte, ici aussi on demande tout à Allah.» Que ce soit une église ne semble rien changer pour elle: "On y a réfléchi en venant, les chrétiens aussi visitent nos mosquées, vous savez, alors pourquoi pas nous... enfin, nous sommes venus en nous disant que nous pouvions nous aussi venir et visiter [une église]. »

La grande majorité des vœux renvoie à des demandes de biens immatériels («imaginaires»), comme la «santé », le «bonheur», la «sérénité » ou la «bonté » (respectivement 39\% des messages, 31, 24 et $22 \%$ ). Viennent ensuite les questions plus matérielles de maison (19\%), d'argent (10\%), de voiture (9\%). Contrairement à d'autres sites, la demande d'enfants semble être peu présente alors même que ce sont majoritairement des femmes qui viennent au monastère. En effet, les exoprates sont principalement des musulmanes au regard des chiffres présentés plus haut. Cette sociographie féminine doit être rapportée à un autre pourcentage effectué dans les bateaux à l'aller. Nous avons décompté près d'un tiers d'hommes. Les hommes «s'évaporent» donc entre le débarcadère et le portillon de sortie du monastère. Revient à ces femmes de gravir la pente raide, d'écrire contre le destin, de transgresser les frontières du religieux, de porter l'espoir qu'autre chose advienne. À elles de dérouler les fils du désir en place de l'Autre. Car, écrire 
un vœu semble être d'abord un acte altruiste, dirigé vers ses proches, sa famille et ses amis. Si les occurrences liées à la déclinaison du moi (ben, bana, benim, etc.) sont présentes dans $18 \%$ des messages étudiés, plus de $52 \%$ contiennent une occurrence liée à la famille, avec une petite préférence pour les mères (anne) et les pères ( $b a b a$ ), les fils (oğlu) et les filles ( $k l z$ ), puis les frères aînés (abey), les sœurs (abla). 60\% des messages contiennent des prénoms qui ne sont pas des signatures. Les femmes sont donc les porteuses de l'espoir des autres. À leur charge de dire un autre rapport à la vérité peu audible pour ceux qui les accueillent (les moines), les protestants évangéliques ou même les autorités religieuses turques. Car, dans le vœu à Aya Yorgi, une forme d'irrévérence à la transcendance s'observe, notamment dans les formes d'élision engagées dans l'acte même de la dédication.

\section{L'oubli du saint}

Le pèlerinage à Büyükada est double: celui de la Saint-Georges (23 avril), puis cinq mois plus tard, celui de la Sainte-Thècle (24 septembre). L'association entre ces deux saints anatoliens n'est pas fortuite: les deux pèlerinages se répondent bel et bien (Couroucli 2009). Lorsqu'un vœu est réalisé à la Saint-Georges, on revient alors remercier en septembre à la Sainte-Thècle. Sauf que

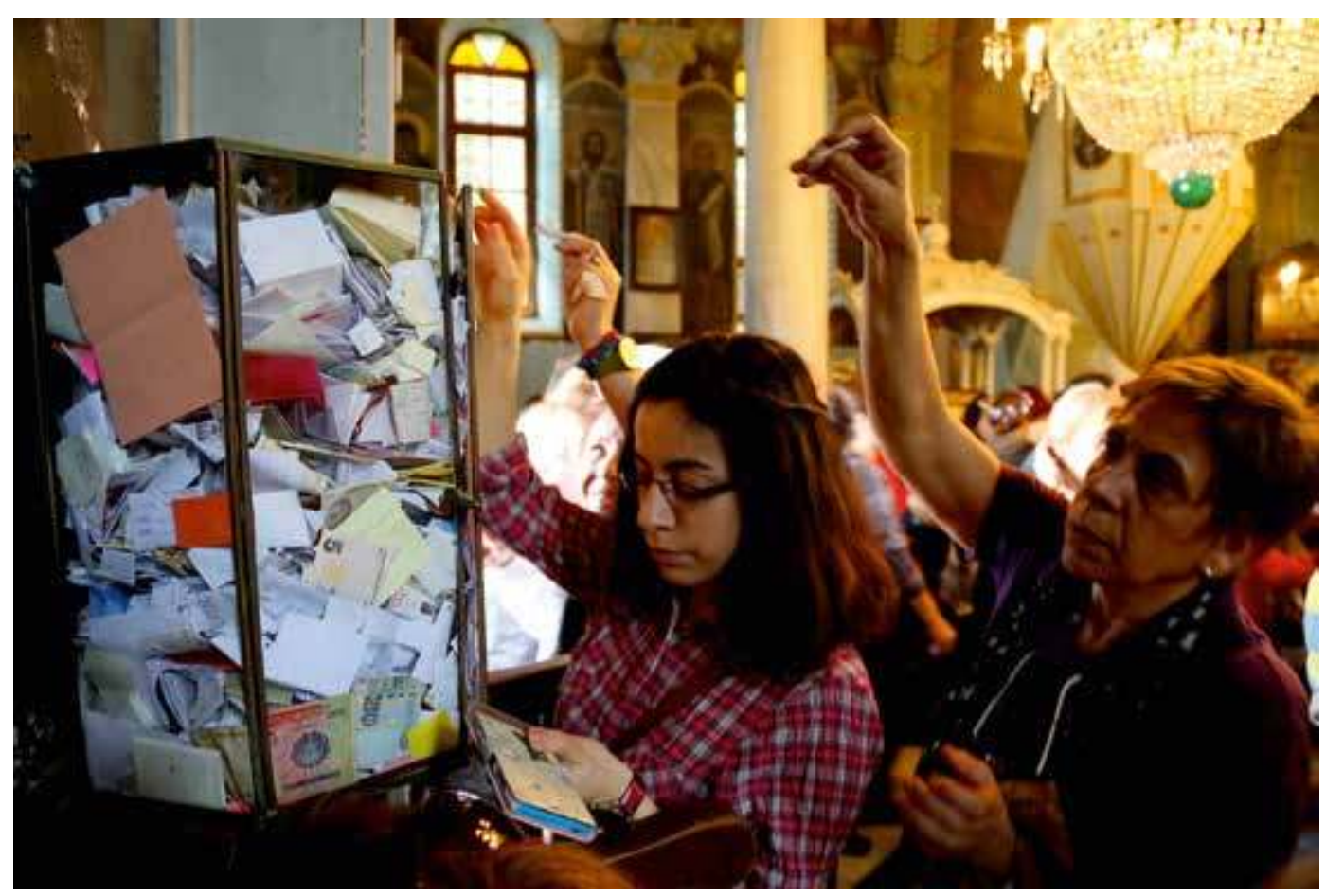

4. Boîte à vœux dans l'église de SaintGeorges 
pour de nombreux pèlerins, le destinataire du remerciement n'est pas nécessairement connu, tout au moins nommé. Les enquêtes conduites le 24 septembre montrent que très peu de gens savent quelle sainte est fêtée ce jour-là. Le 23 avril, il en va généralement de même. Certes les pèlerins se rendent à l'église d'Aya Yorgi, mais est-ce pour autant que leurs vœux s'adressent à lui ? La question mérite d'être posée. Il a été montré ailleurs que les musulmans se rendant dans l'église de Saint-Antoine, à Istanbul, n'écrivaient que rarement au saint sur le livre d'intentions de prière, préférant littéralement s'adresser à Dieu plutôt qu'à ses saints (Fliche 2014). À Büyükada, qui donc agit? Sainte Thècle, saint Georges, Dieu ou autre chose? Sur mille vœux écrits dans les alentours de l'église, récoltés un 23 avril, photographiés et retranscrits, nous comptons seulement trois occurrences de Yorgi (Georges) et 247 occurrences du mot Allah. Saint Georges semble totalement absent. Dieu lui-même ne semble guère présent. Prenons quelques points de comparaison. Benoît Fliche a analysé d'autres pratiques d'écriture votive à Istanbul et a pu constituer des corpus de vœux similaires en volume: le premier pour l'église catholique de Saint-Antoine situé dans le quartier de Péra, le second dans le sanctuaire d'Eyüp (Job) sur les hauteurs de la ville. Dans les deux cas, les visiteurs écrivent leurs vœux soit sur un cahier mis à leur disposition, soit, de manière spontanée, sur des murs. Les occurrences
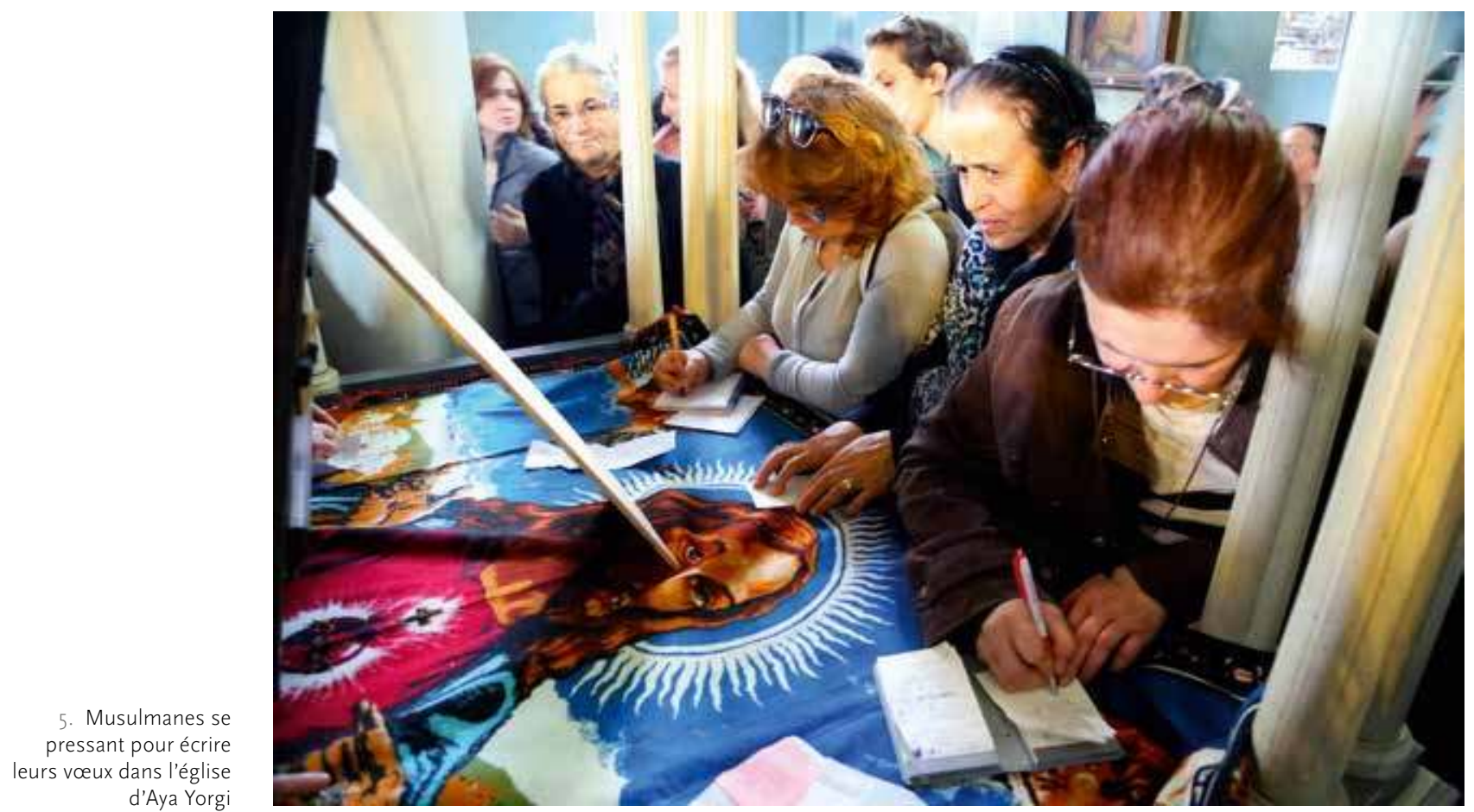
concernant Allah sont de l'ordre de $100 \%$ des messages dans le premier cas contre $77 \%$ dans le second. La personne d'Eyüp est désignée dans 12 messages sur 1000 étudiés, et saint Antoine est invoqué directement dans près d'une centaine de vœux. Que saint Georges soit éludé passe encore - une explication pourrait facilement en être donnée par la logique qui prévaut ailleurs, celle qui voudrait l'absence d'intermédiaire entre Dieu et le croyant - mais à Büyükada, Dieu lui-même semble faire défaut.

\section{Un défaut de Dieu?}

Que veut dire ce manque de Dieu dans les messages votifs écrits? Tout vient contredire cette absence: les bougies, les bobines, la file d'attente durant trois heures sous le soleil, la boîte à vœux que les moines viennent régulièrement vider provoquant parfois de véritables petites émeutes - les femmes craignant de ne pas la voir revenir et rouspétant pour qu'ils les laissent déposer leurs morceaux d'espoir -, les dessins composés en carreaux de sucre ou en brindilles, etc. Que veut dire cette effervescence votive si Dieu et saint Georges sont absents? Notre hypothèse est que cela constitue une résistance à un régime de causalité transcendantale imposée par les clercs. Avec une discrète fermeté, les moines rappellent ce que les croyants sont censés attendre et croire, comme à travers cette pancarte à l'entrée de l'église: «Attacher aux branches des arbres, des ficelles, des fils de nylon, et dans les environs des clefs, des voitures, des maisons, achetés dans une intention votive fait partie des fausses croyances. Lacceptation de vos vœux du côté de DIEU est possible seulement avec un cœur pur et en croyant avec amour. C'est pourquoi, nous souhaitons que nos chers visiteurs n'estiment en aucune façon ces superstitions [croyances vides]. Le monastère d'Aya Yorgi. » De leur côté, les missionnaires évangéliques entendent aussi à leur manière imposer les Écritures, celles qui ne cessent pas de s'écrire. Sans jamais s'adonner à une critique frontale, leur technique de prosélytisme est fondée sur un détournement des codes de la pratique votive à d'autres fins. Ainsi une de leurs pancartes annonçant «les plus belles clefs à vœux, gratuites, pour tout ce que vous voulez», qui désignaient en fait des prières. Il s'agit ni plus ni moins de la manipulation du registre sémiotique votif vers une logique de causalité transcendantale. Ainsi proposent-ils de petites cartes avec des citations de l'Évangile en les mettant à disposition sur un stand à la manière des amulettes déjà évoquées: pour l'amour, le travail, etc. Ironie du sort, les visiteuses n'hésitent pas dès lors à se servir et à réemployer ces cartes pour matérialiser leurs vœux en les suspendant aux arbres, comme si c'était des amulettes. À manipulation, manipulation et demie...

Dans l'église même, en 2011, la «bonne» relation à Dieu et à la causalité divine était rappelée dans une série de pancartes disposées en équilibre sur les murs avec de courts passages des évangiles («Frappez et l'on vous ouvrira», « Heureux les pauvres», etc.). Trois d'entre elles - beaucoup plus explicites sur ce qui était à attendre de Dieu - traduisaient des extraits du livre de Job, notamment quand le prophète dit: «Je suis sorti nu du ventre de ma mère et j'y 
retournerai nu» (Job 1:21), passage qui rejoint les déclinaisons turques des Memento mori. Écrire ou déposer des vœux, c'est finalement faire valoir ainsi un autre régime de causalité.

Les pratiques votives doivent être comprises depuis ce point de l'écriture, écriture à laquelle renvoie littéralement le mot même de vœu puisque l'adak (acte votif), nous rappelle Gokalp (Gokalp 1996), vient de la racine ad - le nom, et désigne ce «qui est destiné à être nommé». L'adak est un acte de nomination: il vient dédicacer un désir, voire le matérialiser, le rendre tangible. Que ce soit sous des formes multiples (carreaux de sucre, brindilles, messages pliés en quatre, cierges, etc.), ces hétérographies sont avant tout des heteropraxeis, autant de pratiques scripturaires qui entendent instaurer autre chose dans les chaînes de détermination.

Dans son séminaire Encore (Lacan 1975), Jacques Lacan distingue quatre régimes de causalité décrits à l'aide de deux couples d'opposition «continue/discontinue ( «cesser» ou «ne pas cesser ») et «détermination/non détermination» («s'écrire / «ne pas s'écrire»). Le possible est «ce qui cesse de s'écrire» : dès lors tout est possible, tout peut advenir puisqu'aucune détermination ne vaut. Le nécessaire est ce qui ne cesse pas de s'écrire. La détermination y est donc entière; il ne peut en être autrement. L'horizon est déjà écrit. L'impossible est le régime où «cela ne cesse pas de ne pas s'écrire». Dès lors rien n'est possible, rien ne peut advenir. Enfin, le contingent est «ce qui cesse de ne pas s'écrire». Dans une rupture avec l'impossible, quelque chose advient qui aurait pu ne pas être et qui, néanmoins, est.

Nous retrouvons la mise en relation de ces quatre régimes de détermination. Alors que le dogme vient dérouler un régime de nécessité - l'écriture divine "ne cesse pas de s'écrire», c'est le destion (kismet) -, que l'horizon bouché du malheur inscrit souvent le sujet dans un impossible - qui «ne cesse pas de ne pas s'écrire», comme l'a défini Lacan dans le séminaire Encore (Lacan 1975) -, et que l'angoisse d'horizons incertains qui «cessent de s'écrire» laissant dès lors le champ libre à tous les possibles, les hétérographies votives «cessent que cela ne s'écrive ». Elles veulent en finir avec les impossibles de la maladie, désirent offrir un point de repère - souvent très matériel - dans le brouillard des possibles. Surtout, et c'est la raison principale de leur condamnation par les orthodoxies religieuses, les hétérographies votives sont autant de ruptures de la nécessité divine. Elles viennent dire que «cela ne s'écrit pas comme il faudrait que cela s'écrive»: qu'un enfant advienne enfin, que l'entrée à l'université soit gagnée, que le visa pour l'ailleurs soit en poche, que quelque chose d'autre s'écrive pour le sujet du désir... et le désir du sujet.

Pratiques de la contingence, ces hétérographies montrent que c'est moins une logique de la sainteté et du miracle - même si celui-ci n'est jamais loin - qu'une logique de l'espoir «sauvage», au sens lévi-straussien, qui prend corps au sommet de l'île de Büyükada. Moins qu'à saint Georges, les pèlerins rendent d'abord visite au lieu sacré. Pour reprendre l'opposition proposée par Dionigi Albera, la logique qui prévaut est celle de la métonymie rituelle; par principes de continuités successives, une relation avec la présence immanente du divin est ainsi maintenue (Albera 2013: 548).

Ce n'est pas le saint mais le lieu de l'espoir qui est prisé, fréquenté. Ainsi, à la question de savoir si le fait d'allumer des cierges revêt un sens particulier, une jeune femme déjà citée plus 


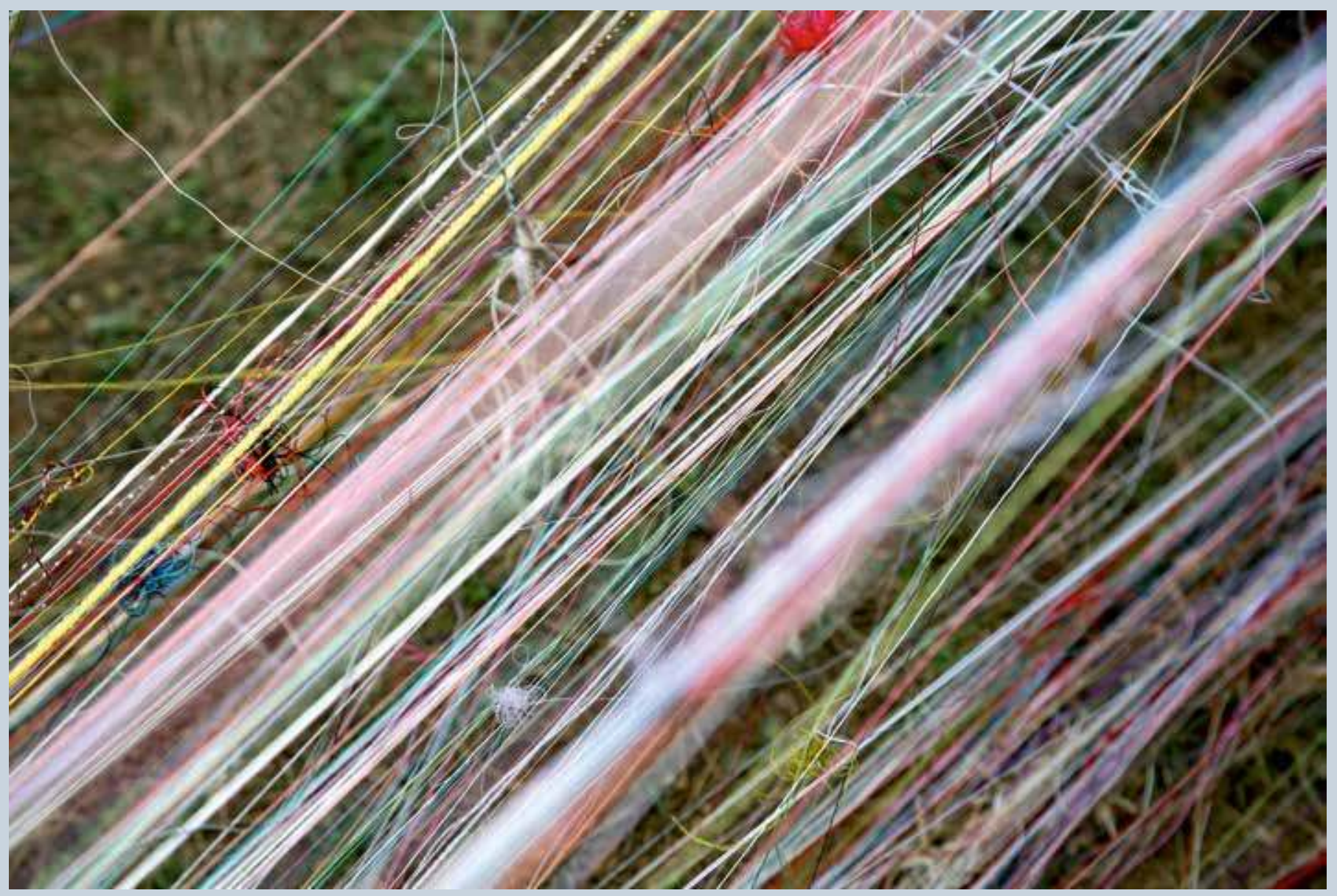

6. Fils votifs entrelacés sur le chemin du monastère 
haut répond: «C'est une tradition. Selon moi, vous pouvez aussi venir sous cet arbre et y faire votre vœu, ça n'a pas d'importance. L'important, c'est votre lien avec le ciel et de souhaiter les choses du fond du cour. Au bout du compte, partout... Si vous souhaitez quelque chose du fond du cœur, il n'y a pas de raison que cela ne se réalise pas. Mais ici (Ama burda), il y a une synergie particulière qui apparemment fait que les vœux se réalisent plus vite.» La fin de cet extrait confirme bien l'efficience du lieu.

Pour conclure, le même espace sacré est traversé et pratiqué par des dynamiques plurielles. Comme le souligne Maria Couroucli, «shared sacra do not imply a shared religious identity: Shared sacra are primarily sacred "places" ( "partager le sacré n'implique pas une identité religieuse partagée : le sacré partagé est avant tout un "lieu" sacré» [tdlr] Couroucli 2012: 45). Larène du pèlerinage de Büyükada concentre une variété de protagonistes qui obéissent à des logiques différentes et potentiellement contradictoires et/ou concurrentielles ${ }^{8}$. Il ressort que les pèlerins ont recours à une large palette d'outils, de gestes et de supports comme si leur usage était «libre de droit». Au fond, ce lieu est attractif, car les gens sont libres de s'exprimer comme ils l'entendent (Eade \& Sallnow 1991), même si cela paraît antinomique ou confus. On remarquera que le lexique rituel commun qui est mobilisé converge vers un dénominateur, celui de l'efficacité rituelle et votive: "parce que ça marche». Se dessine alors une forme d'interreligiosité qui déborde les clivages confessionnels et institutionnels, au grand dam des tenants et gardiens des strictes orthodoxies.

En outre, il nous semble important de revenir sur la notion de la temporalité du vœu, ou plutôt de sa durabilité. Contrairement aux représentations courantes des ex-voto, par exemple en métal et qui sont fait pour durer, force est d'observer qu'ici, c'est l'intention et l'acte votifs qui l'emportent sur le support proprement dit, dans la mesure où la plupart des vœux sont balayés ou jetés le soir même du pèlerinage, ce dont personne ne se soucie particulièrement. En d'autres termes, il semble que le besoin de faire durer ou de conserver une trace du vœu ne soit pas à l'œuvre comme dans d'autres contextes (par exemple dans le catholicisme où les vœux deviennent quasiment des reliques). L'important est que le message soit (hétéro-graphiquement) matérialisé afin que le message passe, en espérant être entendu et exaucé. Si cela advient, le fait de revenir quelques mois plus tard prolonge d'autant la pratique votive, laquelle en alimente de nouvelles par la distribution des carreaux de sucre qui deviennent à leur tour de nouveaux supports d'attentes et de désirs pour d'autres pèlerins. Ces circulations et recyclages de matérialité donnent donc à voir une facette originale et peu connue de la performance votive.

En définitive, qu'est ce qui s'écrit à Aya Yorgi? Le pèlerinage de Saint-Georges n'est qu'une formidable écriture en contre; ces « hétérographies» sont autant de moyens pour restaurer une logique métonymique avec le divin là où devrait prévaloir une logique de séparation, métaphorique, régie par le nécessaire de la prédestination qui ne cesse pas de s'écrire (Albera 2013). Les hétérographies votives ouvrent à une contingence, elles cessent que cela ne s'écrive. Là réside leur efficacité rituelle. À travers elles, plus que de simples attentes, c'est un véritable rapport 
au monde et au divin qui se dessine. L'un des points les plus importants est l'élision du saint, voire de Dieu, qui s'opère dans ce lieu saint et sacré.

L'une des expressions les plus signifiantes de cette religiosité partagée s'incarne dans l'entrelacement des fils multicolores déroulés avec ferveur tout au long du chemin poussiéreux par des pèlerins anonymes, chrétiens ou musulmans. Chaque fil est une écriture de soi. Et le pèlerinage se révèle dès lors comme une vaste machine à tisser des vœux et des désirs, où les femmes, résistantes, font l'espoir des autres.

\section{Notes}

1. Aubin-Boltanski, E. 2007 « Miracles et apparitions de la Vierge au Liban: la preuve par l'Autre», contribution présentée dans le séminaire Anthropologie des dispositifs cultuels, coordonné par Jacques Cheyronnaud et Élisabeth Claverie. [En ligne] : hal.archives-ouvertes.fr/IFPO/halshs-01696870.

2. Citons quelques chapitres récents sur ce pèlerinage à saint Georges: Barkey 2017, Couroucli 2009, Millas 2000, Pinguet 2013

3. Il est aussi sauvé par ce même succès. En effet, ces dernières années, le monastère a connu des recettes suffisantes pour être restauré entièrement (Anastassiadou \& Dumont 2011: 215).

4. Nous définissons «exopraxis» comme une pratique du lieu de l'Autre, qu'elle soit considérée comme orthodoxe ou hétérodoxe. Un musulman qui prie dans une église est exoprate sans être nécessairement hétérodoxe. Toutefois, les espaces-temps d'exopraxis sont aussi des lieux privilégiés d'hétéropraxis (pratiques considérées comme divergentes de la doxa).

5. Dans sa forme actuelle, le pèlerinage est relativement récent, n'ayant pris cette ampleur exoprate qu'à partir de la fin des années 1990. Auparavant, le monastère était surtout un lieu de visite pieuse pour les Grecs orthodoxes. Pour plus d'éléments sur l'historique du lieu, voir Brewer 1830, Millas 2000, Pinguet 2013.

6. Sans analyser cette activité missionnaire, notons cependant son évolution : très marginaux en 2005 2006, ils étaient en concurrence avec des jeunes prosélytes musulmans distribuant des corans édités par le Bureau des Affaires Religieuses lui-même, mais ces derniers ont ensuite disparu.

7. C'est la même tendance qui s'observe au sanctuaire catholique de la Maison de Marie à Éphèse, où des musulmanes déposent leurs vœux sous le tapis de la chapelle (Pénicaud 2014a).

8. Le succès du lieu saint semble avoir atteint un pic en avril 2014. En 2015, le mauvais temps a conduit à une baisse de la fréquentation. Mais en 2016, le pèlerinage a failli être annulé par les autorités à cause d'une rumeur d'attentat par l'organisation auto-proclamée «État Islamique». 


\section{Les auteurs}

Benoît Fliche est anthropologue au CNRS et directeur de l'Institut d'ethnologie méditerranéenne, européenne et comparative (Aix Marseille Université, CNRS, IDEMEC, Aix-en-Provence, France).

Manoël Pénicaud est anthropologue au CNRS (Aix Marseille Université, CNRS, IDEMEC). Spécialisé dans l'étude des pèlerinages, il est aussi l'un des commissaires de l'exposition «Lieux saints partagés » (présentée à Marseille, Paris, Tunis, New York...).

\section{Iconographie}

Image d'ouverture. Étal d'amulettes votives rangées selon les attentes. (C) Manoël Pénicaud.

\section{Références}

Albera, D. 2013 «"Jacob offrit un sacrifice à la montagne" (Genèse, 31): rites et rituels» in D. Albera \& K. Berthelot dir. Dieu, une enquête. Judaïsme, christianisme, islam: ce qui les distingue, ce qui les rapproche. Paris: Flammarion: 457-554.

Albera, D. \& M. Couroucli dir. 2009 Lieux saints partagés entre chrétiens, musulmans et juifs en Méditerranée. Arles: Actes Sud/MMSH.

Albera, D. \& B. Fliche 2009 «Les pratiques dévotionnelles des musulmans dans les sanctuaires chrétiens: le cas d'Istanbul» in D. Albera \& M. Couroucli dir. Religions traversées. Arles: Actes Sud/MMSH: 140-174.

Albera, D. \& M. Pénicaud dir. 2017 Coexistences. Lieux saints partagés en Europe et en Méditerranée. Arles: Actes Sud/MNHI.

Anastassiadou, M. \& P. Dumont 2011 Les Grecs d'Istanbul et le patriarcat ocuménique au seuil du Xxie siècle. Une communauté en quête d'avenir. Paris: Cerf

Barkan, E. \& K. Barkey dir. 2014 Choreographies of Shared Sacred Sites: Religion, Politics, and Conflict Resolution. New York: Columbia University Press.

Barkey, K. 2017 «Le monastère de Saint-Georges et ses visiteurs non chrétiens » in D. Albera \& M. Pénicaud dir. Coexistences. Arles: Actes Sud/MNHI : 62-65.

Brewer, J. 1830 A Residence at Constantinople in the Year 1927. New Haven: Durrie \& Peck.

Certeau, M. de 1990 L'invention du quotidien (T. I, Arts de faire). Paris: Gallimard.

Couroucli, M. 2009 «Saint Georges l'Anatolien, maître des frontières » in D. Albera \& M. Couroucli dir. Religions traversées. Arles, Actes Sud/MMSH: 175-208.

- 2012 «Chthonian Spirits And Shared Shrines: The Dynamics of Place Among Christians and Muslims
1, 3 à 6. (C) Manoël Pénicaud.

2. () Benoît Fliche.

in Anatolia » in Glenn Bowman dir., Sharing the Sacra. New York and Oxford: Berghahn Books.

Dupront, A. 1987 Du Sacré. Croisades et pèlerinages. Images et langages. Paris: Gallimard.

Eade, J. \& M. Sallnow dir. 1991 Contesting the Sacred: The Anthropology of Christian Pilgrimage. Londres et New York: Routledge.

Farra Haddad, N. 2015 «Shared religious rituals and pilgrimage sites: A movement beyond the ChristianMuslim divide » in A. Panagakos dir. Religious Diversity Today: Experiencing Religion in the Contemporary World. Praeger Publishers: Santa Barbara: 89-110.

Fliche, B. 2010 «Les frontières de l'"orthodoxie" et de 1'"hétérodoxie": türbe et églises à Istanbul » in G. Valtchinova dir. Religion, Boundaries, and the Politics of Divine Intervention. Istanbul: ISIS: 81-95.

— 2014 «Une ethnographie de l'indifférence. Les écritures votives de l'église de Saint-Antoine à Istanbul », Ethnologie française 44 : 319-386.

Gokalp, A. 1996 «Les yatır, 1dukut, ou la part de la providence» in J.-L. Bacqué-Grammont \& A. Tibet dir. Cimetières et traditions funéraires dans le monde islamique. Ankara: Türk Tarih Kurumu: 121-124.

Hasluck, F. 2000 [1929] Christianity and Islam Under the Sultans. Istanbul: Isis Press.

Hobart, A. \& T. Zarcone dir. 2017 Pilgrimages and Ambiguity: Sharing the Sacred. Londres: Sean Kingston Publishing.

Lacan, J. 1975 Le Séminaire, Livre XX, Encore (1972-1973). Paris: Seuil.

Lamireau, C. 2009 La poursuite du divin. Écritures votives des lieux de culte catholiques parisiens. Thèse de doctorat, EHESS, Paris. 
Millas, A. 2000 The Princes Islands: A Retrospective Journey. Alimos: Genous Éditions.

Pénicaud, M. 2014a «La Maison de la Vierge à Éphèse. De la fondation à la patrimonialisation d'un sanctuaire international », European Journal of Turkish Studies 19. [En ligne] : ejts.revues.org/4988.

— 2014b «Filmer les pèlerinages et le religieux en mouvement », ThéoRèmes 7 « Capter le rite, filmer le rituel ». DOI : 10.4000/theoremes.673.
Pinguet, C. 2013 Les îles des Princes. Un archipel au large d'Istanbul. Paris: Éditions Empreinte.

Reader, I. 2014 Pilgrimage in the Marketplace. Londres et New York: Routledge.

Valtchinova, G. dir. 2010 Religion and Boundaries: Studies from the Balkans, Eastern Europe, and Turkey. Istanbul: Isis Press.

\section{Pour citer l'article}

Fliche, B. \& M. Pénicaud 2018 « Hétérographies du désir. Pratiques votives au monastère de Saint-Georges (Büyükada, Istanbul)», TechniquesÉCulture 70 « Matérialiser les désirs. Techniques votives», p. 142-161. 